\title{
The membrane molecule RCAS1 induces immune cell apoptosis via the RCAS1-RCAS1R pathway
}

\author{
YUANYUAN ZHANG ${ }^{1 *}$, JIE ZHU $^{2 *}$, XUEJUN HONG $^{3 *}$, YUNLIAN ZHOU $^{1}$, \\ KEMING REN $^{1}$, XIAOLI SHU ${ }^{1}$ and QINGQING WANG ${ }^{4}$ \\ ${ }^{1}$ The Children's Hospital of Zhejiang University School of Medicine, Hangzhou, Zhejiang 310031; \\ ${ }^{2}$ Clinical Laboratory of Zhejiang Hospital, Hangzhou, Zhejiang 310013; ${ }^{3}$ Xuancheng Blood Center, Xuancheng, \\ Anhui 242000; ${ }^{4}$ Institute of Immunology, Zhejiang University, Hangzhou, Zhejiang 310058, P.R. China
}

Received December 4, 2012; Accepted February 15, 2013

DOI: $10.3892 /$ ijmm.2013.1326

\begin{abstract}
Tumor-associated antigen receptor-binding cancer antigen expressed on SiSo cells (RCAS1) has been identified as an estrogen-responsive gene and reportedly acts as a ligand for a putative receptor present in a variety of human cell lines and peripheral lymphocytes, thus leading them to apoptosis. In this study, we investigated the biological function of RCAS1 in vitro in inducing the apoptosis of immune cells. We detected the expression of the RCAS1 receptor (RCAS1R) in the cell lines, and investigated the mechanisms behind the apoptosis induced by RCAS1. HeLa cells were transfected with recombinant adenovirus Ad-RCAS1. RCAS1 induced the apoptosis of activated T cells, K562 cells and phytohemagglutinin (PHA)activated Jurkat cells via the RCAS1-RCAS1R pathway. The expression of RCAS1R was induced. The intracellular overexpression of RCAS1 inhibited the growth of Jurkat and K562 cells. The expression of RCAS1 negatively correlated with the expression of glycogen synthase kinase $3 \beta$ (GSK3 $\beta$ ), but positively correlated with the expression of phosphorylated GSK3 $\beta$ (phGSK3 $\beta$ ). RCAS1 expression was identified as a brown staining pattern in the breast cancer specimens. These findings may provide insight into the mechanisms through which tumor cells escape from immune surveillance.
\end{abstract}

\section{Introduction}

Tumor-associated antigen receptor-binding cancer antigen expressed on SiSo cells (RCAS1) is a type-II membrane protein, expressed in estrogen target organs as well as several other tissues such as the brain, liver and kidney tissues (1).

Correspondence to: Dr Qingqing Wang, Institute of Immunology, Zhejiang University, 388 Yuhangtang Road, Hangzhou, Zhejiang 310058, P.R. China

E-mail: zhang_y_y@sohu.com

${ }^{*}$ Contributed equally

Key words: receptor-binding cancer antigen expressed on SiSo cells, RCAS1-RCAS1R, pathway, apoptosis, immune cell
Previous studies have shown that RCAS1 is expressed in a variety of malignancies, such as breast, ovarian, prostate and hepatocellular carcinoma (2-5). The RCAS1 protein acts as a ligand for a putative receptor present in various human cells, including normal peripheral lymphocytes, such as T, B and natural killer (NK) cells. RCAS1 inhibits the growth of receptor-expressing cells both in vitro and in vivo and induces apoptotic cell death through the activation of caspase- 3 and the collapse of mitochondrial transmembrane potential $(6,7)$. Therefore, it has been generally accepted that RCAS1 is involved in the immune escape of tumor cells. Immunohistochemical studies of tissue samples have revealed that RCAS1 expression significantly correlates with poor overall survival in patients with a variety of malignancies (8-10). However, the possible mechanism behind the RCAS1-induced cell apoptosis has not yet been elucidated.

In our previous studies, we induced the expression and purification of glutathione S-transferase (GST)-RCAS1 fusion protein (11), and reported that RCAS1 promoted the tumor growth and metastasis of 4T1 murine mammary carcinoma cells (12). In this study, we used the recombinant adenovirus RCAS1 (Ad-RCAS1) and GST-RCAS1 fusion protein, to investigate the apoptosis of $\mathrm{T}$ lymphocytes and immune cells derived from leukemia cell lines induced by RCAS1. We detected the expression of the RCAS1 receptor (RCAS1R) in the cell lines, and investigated the mechanism behind the apoptosis induced by RCAS1. We used immunohistochemical analysis to examine the expression of this molecule in human breast tissues.

\section{Materials and methods}

Reagents. Lipofectamine 2000 was obtained from Invitrogen (Carlsbad, CA, USA). GST monoclonal antibody (MoAb) was purchased from Kangchen Biotech Co. (Shanghai, China). The B-PER GST Fusion Purification kit was purchased from Pierce Biotechnology (Rockford, IL, USA). Mouse GAPDH MoAb, donkey anti-rabbit IgG $(\mathrm{H}+\mathrm{L})$ horseradish peroxidase (HRP)-, donkey anti-goat IgG $(\mathrm{H}+\mathrm{L}) \mathrm{HRP}$ - and goat anti-mouse IgG (H+L) HRP-conjugated antibodies were all from Cell Signaling Technology, Inc. (Danvers, MA, USA). Goat polyclonal antibodies (PoAbs) to RCAS1 (N18 and C20; sc-23396 and sc-23394, respectively; Santa Cruz 
Biotechnology, Inc., Santa Cruz, CA, USA), rabbit PoAbs to glycogen synthase kinase $3 \beta$ (GSK $3 \beta$ ) and phospho-Ser9GSK $3 \beta$ (BioVision Technologies, Exton, PA, USA) were commercially purchased. RCAS1 primer was synthesized by Shanghai Sangon Biological Engineering Technology and Services Co., Ltd. (Shanghai, China). This study was carried out in accordance with the Declaration of Helsinki. All the data presented and materials used in this manuscript were approved by the Ethics Committee of the Children's Hospital of Zhejiang University School of Medicine, Hangzhou, China.

Cell culture and treatment. A293 (human embryonic kidney), HeLa (adenocarcinoma), K562 (chronic myelogenous leukemia), Jurkat (acute T cell leukemia), THP1 (acute monocytic leukemia) and E003 (acute B cell leukemia) cells were from the American Type Culture Collection (Manassas, VA, USA). All the cells were grown in RPMI-1640 supplemented with $10 \%(\mathrm{v} / \mathrm{v})$ fetal calf serum, $4.5 \mathrm{~g} / \mathrm{l} \mathrm{D}$-glucose, non-essential amino acids (100 $\mu \mathrm{M}$ each), $100 \mathrm{U} / \mathrm{ml}$ penicillin, $100 \mu \mathrm{g} / \mathrm{ml}$ streptomycin and $2 \mathrm{mM}$ glutamine at $37^{\circ} \mathrm{C}$ in a $5 \% \mathrm{CO}_{2}$ atmosphere. HeLa cells were transfected with the Ad-RCAS1 and Ad-LacZ vectors, using Lipofectamine 2000 according to the manufacturer's instructions. Following transfection, RT-PCR and western blot analysis were carried out to determine the expression of RCAS1, $\beta$-actin or GAPDH, and the effectiveness of the assay was determined by the negative control. K562, Jurkat, THP1 and E003 cells were cultured in 6 -well flat bottom plates $\left(5 \times 10^{5}\right.$ cells/ well), and then Ad-RCAS1 $\left[5 \times 10^{7}\right.$ plaque forming units (PFU), multiplicity of infection (MOI) 100] and Ad-LacZ (5x10 7 PFU, MOI 100) were added to the medium. After $24 \mathrm{~h}$ of culture, the cells were harvested for apoptosis assay.

Amplification of recombinant adenovirus Ad-RCAS1 and Ad-LacZ. Recombinant adenovirus Ad-RCAS1 and Ad-LacZ were gifts from Professor Cao (Second Military Medical University, Shanghai, China). For recombinant adenovirus multiplication, A293 cells with $80 \%$ fusion were replenished with fresh medium and subsequently infected with $100 \mu \mathrm{l}$ of Ad-RCAS1 or Ad-LacZ. After the cytopathic effect was achieved (approximately 24 to $72 \mathrm{~h}$ ), the cells were collected and washed 3 times with PBS, then metered in a total volume of $200 \mu \mathrm{l}$. After 6 freeze-thaw cycles, the cells were centrifuged at $5,000 \mathrm{rpm}$ at $4^{\circ} \mathrm{C}$ for $15 \mathrm{~min}$, and the virus was released into the supernatants (approximately $200 \mu \mathrm{l}$ ). Following amplification, all viruses were mixed and filtered through a Gelman syringe filter $(0.2 \mu \mathrm{m})$ for the experiments. Virus titer $(\mathrm{PFU} / \mathrm{ml})$ was determined by a $50 \%$ tissue culture infective dose (TCID50) assay (13).

Isolation and culture of $T$ cells and $C D 4^{+} T$ cells. Peripheral blood was obtained from volunteers (Blood Center, Zhejiang, China). Peripheral blood mononuclear cells (PBMCs) were obtained by Percoll density gradient centrifugation using lymphocyte separation medium (Henxin Bio Co., Shanghai, China) from human peripheral blood. $\mathrm{T}$ cells $\left(1 \times 10^{8}\right)$ were purified using MACS Columns (Miltenyi Biotec). $\mathrm{CD}^{+}{ }^{+} \mathrm{T}$ cells and $\mathrm{CD}^{-}{ }^{-} \mathrm{T}$ cells were acquired. The PBMCs and $\mathrm{CD}^{+}{ }^{+} \mathrm{T}$ cells were then cultured in RPMI-1640 in 6-well flat bottom plates (Orange Scientific), supplemented with $200 \mathrm{U} / \mathrm{ml}$ interleukin (IL)-2. The old medium was replaced with fresh medium every
2 days. On day 7, the cells were treated with $1 \mu \mathrm{g} / \mathrm{ml}$ phytohemagglutinin (PHA), then cultured for $24 \mathrm{~h}$. On day 8, the cells were randomly divided into 3 groups: controls, Ad-RCAS1 and Ad-LacZ; after $24 \mathrm{~h}$ of culture, the cells were harvested for apoptosis assay (propidium iodide and Annexin V staining).

Apoptosis assay. The cells were washed, resuspended in staining buffer, and stained with propidium iodide and Annexin V, according to the manufacturer's instructions. Stained cells were analyzed using a FACSCalibur (BectonDickinson). The Annexin V-FITC Apoptosis Detection kit was purchased from BioVision Technologies.

RT-PCR analysis. Total RNA was extracted from the cultured cells using TRIzol reagent (Dingguo Biotech Co., Beijing, China). Total RNA (1 $\mu \mathrm{g})$ from each sample was reverse transcribed using a Reverse Transcription System kit (MBI Fermentas, Vilnius, Lithuania) in a total volume of $20 \mu \mathrm{l}$. cDNA synthesized from mRNA was quantified by PCR using specific primers as follows: RCAS1 sense, 5'-GCGAATTCTT ATGAAAGTTTCACACC-3' and antisense, 5'-GTGGATCC ATGGCCATCACCCAGTTT-3', with a predicted PCR product of $642 \mathrm{bp} ; \beta$-actin sense, 5'-CCTAGAAGCATTT GCGGTGG-3' and antisense, 5'-GAGCTACGAGCTT GACG-3', with an expected PCR product of $402 \mathrm{bp}$. Cycling conditions were $30 \mathrm{sec}$ of denaturation at $94^{\circ} \mathrm{C}, 30 \mathrm{sec}$ of annealing at $56-60^{\circ} \mathrm{C}$ and $60 \mathrm{sec}$ of extension at $72^{\circ} \mathrm{C}$. The relative amount of mRNA was determined using Quantity One software (Bio-Rad, Hercules, CA, USA).

Western blot analysis. Cells were lysed in lysis buffer (Cell Signaling Technology, Inc.) for $30 \mathrm{~min}$ on ice. A BCA kit (Cat. no. 23225; Pierce Biotechnology) was then used to determine the concentration of the lysates. Cell extracts were separated by $10 \%$ sodium dodecyl sulfate-polyacrylamide gel electrophoresis and transferred onto polyvinylidene difluoride membranes. The blot was probed with RCAS1 (C20 and N18), GSK $3 \beta$ and phosphorylated GSK3 $\beta$ (phGSK $3 \beta$ ) PoAbs and GAPDH MoAb. Signals were developed using an enhanced chemiluminescence detection reagent. The relative protein level was determined with band intensities compared with GAPDH using the Quantity One software (Bio-Rad).

Receptor detection. The binding of GST-RCAS1 to RCAS1 receptor (RCAS1R)-expressing cells was determined by fluorescence-activated cell sorting (FACS) analysis. Briefly, after 3 washes with PBS, the K562, Jurkat or PHA-treated Jurkat cells $\left(3 \times 10^{5}\right)$ were incubated in $1 \mathrm{ml}$ PBS, with the addition of $10 \mu \mathrm{l}$ GST-RCAS1 $(10 \mu \mathrm{g})$ fusion protein, GST protein $(10 \mu \mathrm{g})$ and PBS, respectively, and incubated for $30 \mathrm{~min}$ at $4^{\circ} \mathrm{C}$. After 3 washes, GST MoAb was added, followed by incubation for $30 \mathrm{~min}$ at room temperature. After 3 washes, the cells were labeled with anti-mouse IgG conjugated with fluorescein isothiocyanate (FITC) and incubated for $10 \mathrm{~min}$ at room temperature in the dark. After another 3 washes, the cells were resuspended in a final volume of $1 \mathrm{ml}$ of PBS and acquired on a FACSCalibur (Becton-Dickinson). The experiments were repeated on three separate occasions and the percentage and fluorescence intensity of FITC-positive cells were detected by FACS. 
3-(4,5-Dimethylthiazol-2-yl)-2,5-diphenyltetrazolium bromide (MTT) assay. K562 and Jurkat cells were cultured in 96-well plates at a density of $1 \times 10^{4}$ cells/well in RMPI-1640 supplemented with $5 \%$ fetal calf serum (FCS) in the absence or presence of Ad-RCAS1 or Ad-LacZ $\left(0.625 \times 10^{5}-40 \times 10^{5} \mathrm{PFU}\right.$, MOI 12.5-400). After $48 \mathrm{~h}$ of incubation, $20 \mu \mathrm{l}$ of MTT $(5 \mathrm{mg} / \mathrm{ml})$ (Sigma) were added to each well. The mixture was incubated for an additional $4 \mathrm{~h}$ and then centrifuged at $3,000 \mathrm{rpm}$ at $4^{\circ} \mathrm{C}$ for $10 \mathrm{~min}$, and the supernatant was discarded. The precipitate was dissolved in $150 \mu \mathrm{l}$ of DMSO and the absorbance values were read at $570 \mathrm{~nm}$ with a microplate reader (Molecular Devices, Palo Alto, CA, USA).

Co-immunoprecipitation. Cell lysates of K562 cells transfected with Ad-RCAS1 (300 $\mu \mathrm{g} /$ tube) were mixed with RCAS1 PoAb, control or GSK3 $\beta$ PoAb, and incubated for $6 \mathrm{~h}$. Then a $3 \mathrm{X}$ reaction volume of Protein G Plus Agarose $(60 \mu \mathrm{l})$ was added, followed by incubation for another $6 \mathrm{~h}$. The cells were then washed 3 times in PBS, followed by the addition of the buffer $(120 \mu \mathrm{l})$, and subjected to western blot analysis.

Histological examination of breast tissue. Different breast tissues for histological examination were collected from the Women's Hospital School of Medicine, Zhejiang University. Immunohistochemical analysis was performed by Chaoying Bio Co. (Shanxi, China). Slides (5- $\mu \mathrm{m}$-thick) were deparaffinized, rehydrated and rinsed in distilled water. Endogenous peroxidase activity was blocked by $10 \mathrm{~min}$ of incubation in $3 \%$ $\mathrm{H}_{2} \mathrm{O}_{2}$ at room temperature. The slides were then rinsed and immersed in boiling citrate buffer ( $\mathrm{pH}$ 6.0) in a microwave oven with 3 changes of buffer every $5 \mathrm{~min}$. For the immunolocalization of RCAS1, the slides were treated with the goat PoAbs to RCAS1 (N18 and C20; dilution 1:100, 1:50) in a moist chamber overnight. The slides were subsequently rinsed in TBS buffer (pH 7.6) and incubated with secondary antibody [donkey antigoat $\mathrm{IgG}(\mathrm{H}+\mathrm{L}) \mathrm{HRP}$ ] (dilution 1:500, 1:250) for $45 \mathrm{~min}$ at $37^{\circ} \mathrm{C}$. Visualization was performed using 3,3'-diaminobenzidine (DAB) as a chromogen for $10 \mathrm{~min}$ at room temperature. The sections were counterstained with hematoxylin and mounted in Glycergel. We assumed that positive reactivity for RCAS1 occurred when we observed a brown staining pattern.

Statistical analysis. All experiments were repeated 2 or 3 times. Statistical analysis was carried out using the Student's t-test. A P-value $<0.05$ was considered to indicate a statistically significant difference.

\section{Results}

Identification of recombinant adenovirus Ad-RCASI. To determine whether RCAS1 expression affects cellular apoptosis in vitro, we amplified recombinant adenovirus Ad-RCAS1. Ad-LacZ was amplified as the control. In this study, we amplified $5 \mathrm{ml}$ Ad-RCAS1 and Ad-LacZ, respectively. TCID50 assay showed that Ad-RCAS1 and Ad-LacZ titers were $4.8 \times 10^{9}$ and $4.2 \times 10^{9} \mathrm{PFU} / \mathrm{ml}$, respectively, then adjusted to a final titer of $4 \times 10^{9} \mathrm{PFU} / \mathrm{ml}$. HeLa cells were then transfected with Ad-RCAS1 or Ad-LacZ. The expression of RCAS1 was identified by RT-PCR and western blot analysis (Fig. 1A-C). Semi-quantitative RT-PCR analysis showed that the mRNA


Figure 1. Identification of Ad-RCAS1 by RT-PCR and western blot analysis in HeLa cells transfected with Ad-RCAS1. ${ }^{*} \mathrm{P}<0.05$. HeLa cells were transfected with Ad-RCAS1, or Ad-lacZ as the mock control using Lipofectamine 2000. The expression of RCAS1 in the Ad-RCAS1-transfected HeLa cells (HeLa/ Ad-RCAS1) was detected by (A and B) RT-PCR and (C) western blot analysis with anti-N18 and anti-C20 antibody.The experiment was carried out 3 times and 1 representative result is shown.

expression of RCAS1 in the HeLa cells transfected with Ad-RCAS1 was higher than that in the HeLa cells transfected with Ad-LacZ or in the parental HeLa cells. Simultaneously, the density of the $32 \mathrm{kDa}$ bands detected by western blot analysis using PoAb to RCAS1 (C20 and N18) was relatively higher in the cell lysates of the HeLa cells transfected with Ad-RCAS1 than that in the cell lysates of the HeLa cells transfected with Ad-LacZ or in the parental HeLa cells.

Detection of apoptosis in T lymphocytes and immune cells derived from leukemia cell lines following treatment with RCAS1. To investigate whether RCAS1 can induced the apoptosis of $\mathrm{T}$ cells, we examined the percentage of apoptotic cells by propidium iodide and Annexin $\mathrm{V}$ staining. Twenty-four hours after the transfection of $\mathrm{T}$ cells isolated from peripheral blood with Ad-RCAS1, Ad-LacZ or the blank control, a small number of apoptotic cells was observed and no significant differences were detected in the $\mathrm{T}$ cells transfected with Ad-RCAS1 compared with those transfected with Ad-LacZ or the blank control (data not shown). After the T cells were stimulated with IL-2 (200 IU/ml) and PHA $(1 \mu \mathrm{g} / \mathrm{ml})$, we found that the apoptotic value of Ad-RCAS1 in the activated T cells was higher than that of the Ad-LacZ or the blank control 

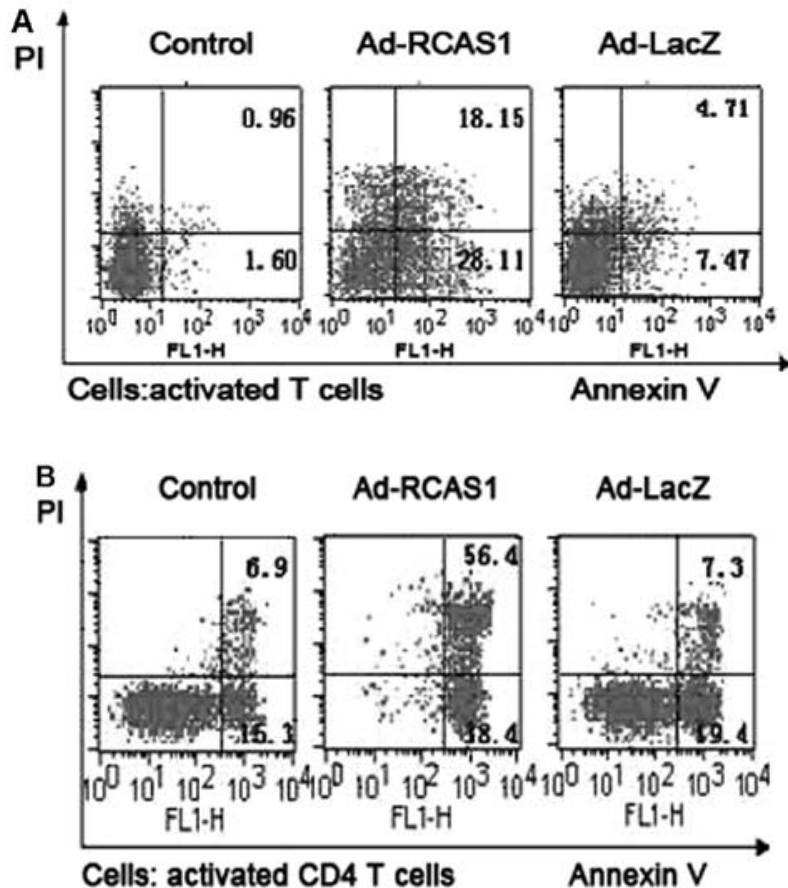

Figure 2. Effect of RCAS1 on activated $\mathrm{T}$ cell and $\mathrm{CD} 4^{+} \mathrm{T}$ cell apoptosis in vitro. (A) Activated $\mathrm{T}$ cell and (B) $\mathrm{CD}^{+} \mathrm{T}$ cell apoptosis detected by FACS with propidium iodide (PI) and Annexin V staining. Activated T cells and $\mathrm{CD}^{+} \mathrm{T}$ cells were transfected with Ad-RCAS1, Ad-LacZ and medium, and harvested for apoptosis assay (PI and Annexin V staining). The experiment was carried out 3 times and 1 representative result is shown.

group (Annexin V-positive cells 46.26, 12.18 and 2.56\%, respectively) (Fig. 2A). We examined the effect of RCAS1 on activated $\mathrm{CD}^{+} \mathrm{T}$ cell apoptosis. As shown in Fig. 2B, an increased number of apoptotic cells was observed in the activated $\mathrm{CD}^{+} \mathrm{T}$ cells transfected with Ad-RCAS1 compared with those transfected with Ad-LacZ or the blank control (Annexin V-positive cells 94.8, 56.7 and 23.2\%, respectively). These results suggest that RCAS1 overexpression induces the apoptosis of activated $\mathrm{T}$ cells and $\mathrm{CD} 4^{+} \mathrm{T}$ cells.

Furthermore, using the same method, we examined whether RCAS1 affects the apoptosis of immune cells derived from leukemia cell lines, such as Jurkat, K562, THP1 and E003 cells. K562 cells exhibited higher levels of apoptosis following Ad-RCAS1 treatment, compared with the cells treated with Ad-LacZ or the blank control (Annexin V-positive cells 36.7, 18.8 and $6.8 \%$, respectively) (Fig. 3A). However, RCAS1 had no effect on the apoptosis of THP1 and E003 cells (data not shown). For the Jurkat cells (acute T cell leukemia), the apoptotic value of Ad-RCAS1, Ad-LacZ and the blank control group was 31.2, 20.2 and $13.2 \%$, respectively (Fig. 3B). The Ad-RCAS1 group had the highest rate of apoptosis, whereas the blank control group had the lowest rate. As shown in Fig. 3C, an increased number of apoptotic cells was observed in the Jurkat cells stimulated with PHA $(1 \mu \mathrm{g} / \mathrm{ml})$, and the difference in the number of apoptotic cells between the Ad-RCAS1 and Ad-LacZ or the blank control group was significant.

RCAS1 receptor detection. In our previous study, we induced the expression and purification of GST-RCAS1 fusion protein (11). In this study, using the GST-RCAS1 fusion

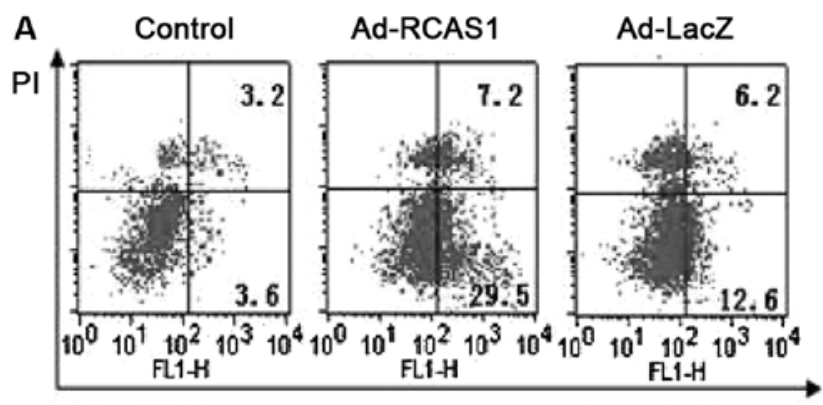

Cells: K562

Annexin V
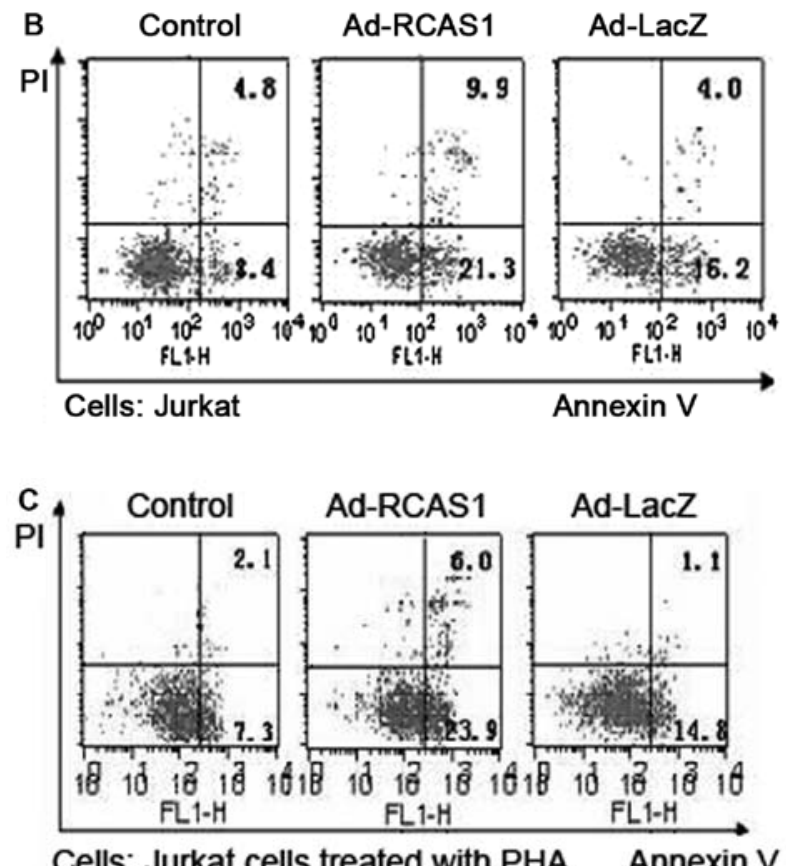

Figure 3. Apoptosis of Jurkat and K562 cells induced by RCAS1. The apoptosis of (A) K562, (B) Jurkat and (C) PHA-activated Jurkat cells was detected by FACS with propidium iodide (PI) and Annexin V staining. K562, Jurkat, PHA-activated Jurkat cells were transfected with Ad-RCAS1, Ad-LacZ and medium, and harvested for apoptosis assay (PI and Annexin V staining). The experiment was carried out 3 times and 1 representative result is shown.

protein, we examined whether RCAS1R is expressed on the cell membrane of Jurkat, K562, THP1 and E003 cells. In the K562 cells, RCAS1R expression was detected and there was a significant difference between the GST-RCAS1 group and the GST or control group; however, the percentage of FITC-positive cells was not very high (Fig. 4A). Of note, a low expression of RCAS1R was detected in the THP1, E003 (data not shown) and Jurkat cells (Fig. 4B). As shown in Fig. 4C, RCAS1 expression in the Jurkat cells co-cultured with GST-RCAS1 increased significantly following stimulation with PHA $(1 \mu \mathrm{g} / \mathrm{ml})$ compared with the cells co-cultured with GST or the blank control (FITC-positive cells 43.98, 1.59 and $4.07 \%$, respectively). These results suggest that RCAS1R is not expressed in Jurkat cells; however, following stimulation with PHA, a moderate upregulation of its expression can be induced.

RCAS1 inhibits $K 562$ and Jurkat cell proliferation. We used MTT assay to examine whether RCAS1 affects the 


\section{A RCAS1 receptor detection in $\mathrm{K} 562$ cells}
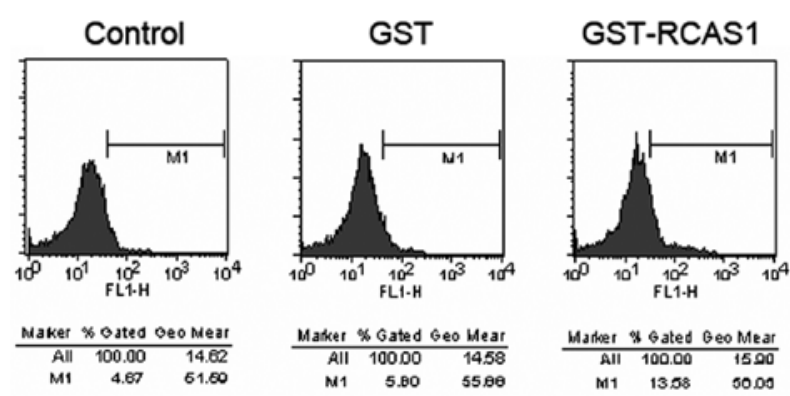

B

RCAS1 receptor detection in Jurkat cells
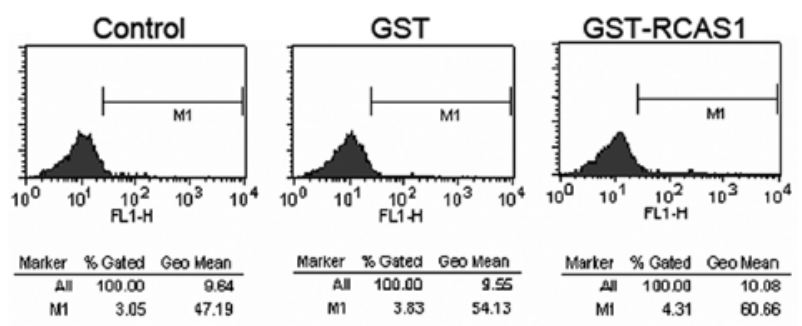

\section{RCAS1 receptor detection in PHA treated Jurkat cells}
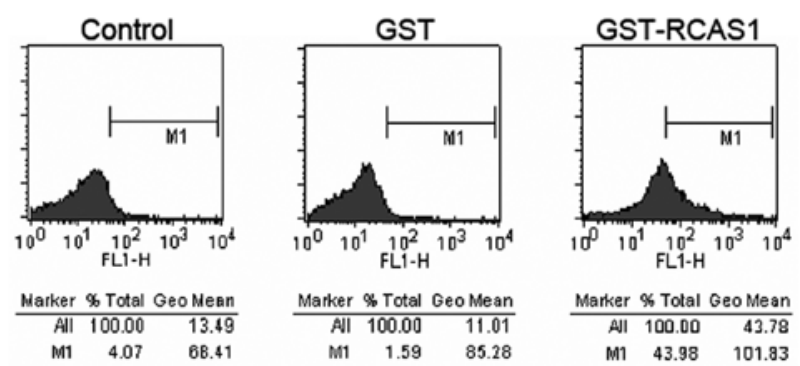

Figure 4. Putative RCAS1 receptor detection by FACS using GST-RCAS1 fusion protein. Cell surface expression of RCAS1 in (A) K562, (B) Jurkat and (C) PHA-treated Jurkat cells determined by FACS using GST-RCAS1 fusion protein. GST protein or a blank were used as controls. The experiment was carried out 3 times and 1 representative result is shown.

proliferation of K562 and Jurkat cells. Fig. 5A and B shows the inhibitory effect of RCAS1 on the proliferation of K562 and Jurkat cells at $48 \mathrm{~h}$ post-transfection at different MOIs of Ad-RCAS1 and Ad-LacZ. There was a significant difference in the cell viability between Ad-RCAS1 and Ad-LacZ in a MOI-dependent manner, indicating that RCAS1 inhibited K562 and Jurkat cell growth in vitro.

Correlation between overexpression of RCAS1 and GSK3 $\beta$ or phGSK3 $\beta$. Using western blot analysis and co-immunoprecipitation, we examined whether GSK3 $\beta$ plays a role in the signaling pathway of cell apoptosis induced by RCAS1. When the MOI of Ad-RCAS1 was doubled in the K562 cells, the expression of RCAS1 in the K562 cells increased, but the expression of GSK $3 \beta$ decreased, and the expression of phGSK3 $\beta$ increased (Fig. 6A). No significant difference was observed in the K562 cells transfected with Ad-LacZ (Fig 6B), indicating that the overexpression of RCAS1 in the K562 cells resulted in the altered expression of GSK $3 \beta$ and phGSK3 $\beta$. Our data suggest that the expression of RCAS1 negatively correlates

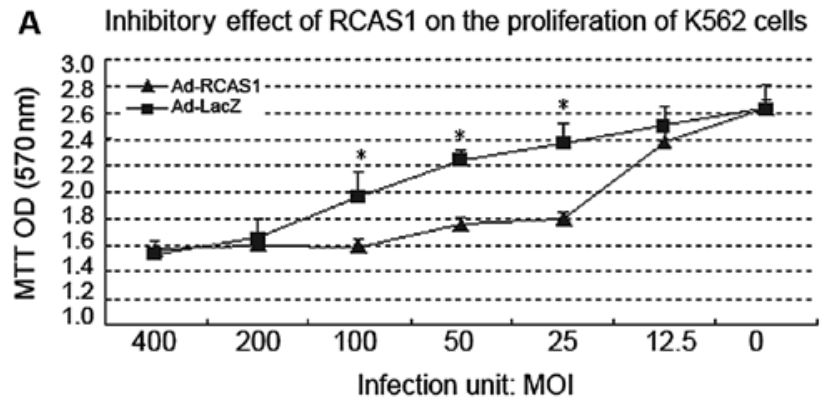

B Inhibitory effect of RCAS1 on the proliferation of Jurkat cells

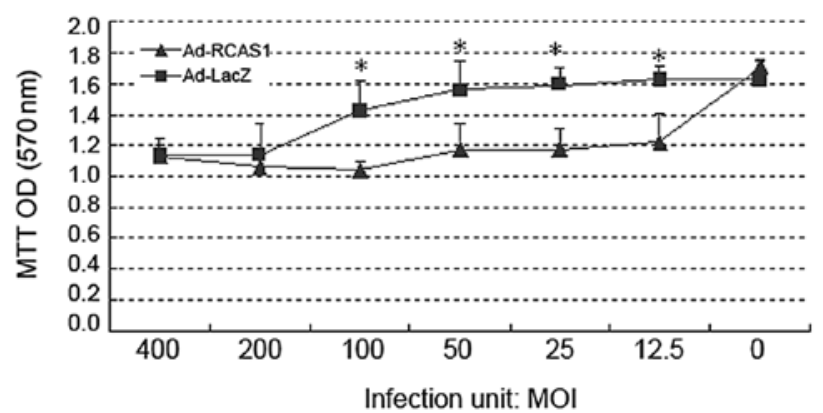

Figure 5. Effect of RCAS1 on (A) K562 and (B) Jurkat cell viability examined by MTT assay in vitro. After co-culture with Ad-RCAS1 (Ad-LacZ as the mock control) at different MOIs (0, 12.5, 25, 50, 100, 200 and 400), cell proliferation was evaluated. Data are presented as the means \pm standard deviation. Similar results were obtained in 3 independent experiments. ${ }^{*} \mathrm{P}<0.05$.



B RCAS1 influencing the expression of GSK3 $\beta$ in K562 cells



Figure 6. Western blot analysis of the correlation between RCAS1 and GSK $3 \beta$ or phGSK3 $\beta$. K562 cells were transfected with (A) Ad-RCAS1 or (B) Ad-LacZ at an MOI from 0 to 160, and the cell lysates were analyzed by western blot analysis using RCAS1, GSK3, or phGSK3 $\beta$ polyclonal antibody and GAPDH monoclonal antibody (MoAb) as the control. The experiment was carried out 3 times and 1 representative result is shown. 


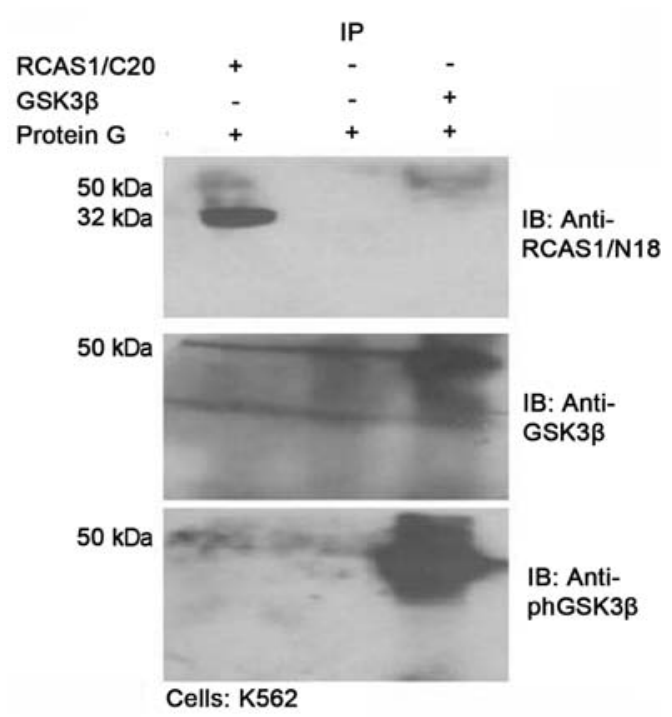

Figure 7. Results of the co-immunoprecipitation assay. Lysates of K562 cells were immunoprecipitated with either RCAS1/C20 polyconal antibody (PoAb) or GSK $3 \beta$ PoAb and protein $G$ as the control. The immunoprecipitated proteins were detected with RCAS1/N18 PoAb, GSK $3 \beta$ PoAb and phGSK $3 \beta$ PoAb. The experiment was carried out 3 times and 1 representative result is shown.

with the expression of GSK $3 \beta$, but positively correlates with the expression of phGSK3 $\beta$. Furthermore, we determined whether RCAS1 binds to GSK3 $\beta$, leading to the phosphorylation of GSK3 $\beta$ by using co-immunoprecipitation. RCAS1 was not detected in the immunoprecipitates of K562 cells transfected with Ad-RCAS1, which were precipitated with anti-GKS3 $\beta$ and anti-phGKS3 $\beta$ antibody (Fig. 7). This result demonstrates that RCAS1 does not directly bind to GSK3 $\beta$.

RCASI expression identified as a brown staining pattern in breast cancer specimens. In our previous study, we reported that RCAS1 promoted tumor growth and metastasis in 4T1 murine mammary carcinoma cells (12). In this study, we collected different types of breast tissue from the Women's Hospital School of Medicine, Zhejiang University for histological examination. For the immunolocalization of RCAS1 the specimens were treated RCAS1 (N18 and C20) PoAb. Immunohistochemical analysis confirmed that the breast cancer tissue expressed RCAS1; however, the normal breast tissue did not express RCAS1 (Fig. 8).

\section{Discussion}

RCAS1 is a type-II membrane protein, expressed in estrogen target organs, as well as in various malignancies. Nakashima et al (6) showed that RCAS1 was a transmembrane molecule, and that activated $\mathrm{T}$ cells expressed a putative RCAS1R, and apoptosis was induced after the binding between RCAS1 and RCAS1R. However, other studies have provided conflicting results without showing any significant correlation. Engelsberg el al (14) showed that RCAS1 was predominantly located on Golgi and that RCAS1 was not able to induce apoptosis, also confirmed by the study of Reimer et al (15). In our study, we constructed the GST-RCAS1 fusion protein and recombinant adenovirus Ad-RCAS1 to examine the biological function of RCAS1 in vitro in inducing the apoptosis of immune cells.

To determine whether RCAS1 expression affects cell apoptosis in vitro, we amplified the recombinant adenovirus Ad-RCAS1. Prior to its use, Ad-RCAS1 was analyzed by RT-PCR and western blot analysis. HeLa cells are a cervical adenocarcinoma cell line, which does not express RCAS1 (6). In our study, HeLa cells were transfected with Ad-RCAS1, thus inducing the expression of RCAS1 in the HeLa cells. Cells were also transfected with Ad-LacZ as the controls. Using RT-PCR, we found that RCAS1 was expressed in the HeLa cells transfected with Ad-RCAS1, the HeLa cells transfected with Ad-LacZ and in the parental HeLa cells; this results is contradictory to the results of other studies (6). Semi-quantitative RT-PCR analysis showed that the mRNA expression of RCAS1 in the HeLa cells transfected with Ad-RCAS1 was higher than that in the HeLa cells transfected with Ad-LacZ or in the parental HeLa cells; this difference was statistically significant. Simultaneously, $32 \mathrm{kDa}$ bands were detected by western blot analysis using RCAS1 PoAb (C20 and N18) in the 3 groups of HeLa cells, but the density of the bands was upregulated in the HeLa cells transfected with Ad-RCAS1 compared to the HeLa cells transfected with Ad-LacZ or in the parental HeLa cells.

After amplifing recombinant adenovirus Ad-RCAS1 and Ad-LacZ, we determined and corrected the virus titer. Due to the biological effects of adenovirus on cells, a consistent concentration should be used for experiments. The virus titer

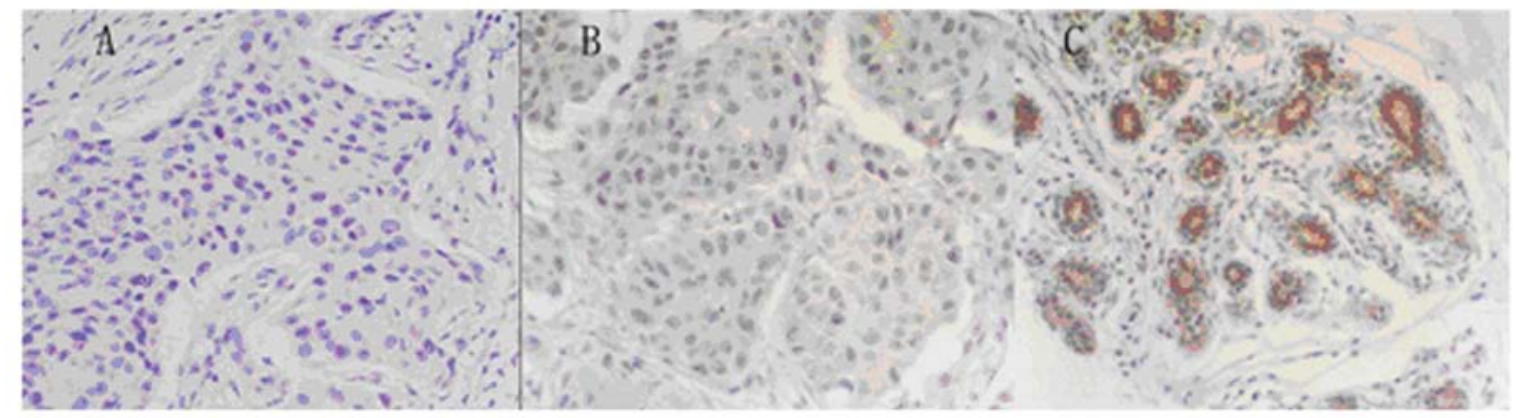

Figure 8. Detection of RCAS1 expression in different breast tissue specimens. (A) In the normal breast tissue, RCAS1 expression was not detected. Immnohistochemical detection of (B) RCAS1/C20 and (C) RCAS1/N18 in breast cancer tissue. Brown staining indicates RCAS1 expression. Original magnification, $\mathrm{x} 200$. 
(PFU/ml) was determined by TCID50 assay. Ad-RCAS1 and Ad-LacZ titer were then adjusted to the same concentration.

In our study, we separated peripheral blood lymphocytes from human peripheral blood as the cellular model to investigate whether RCAS1 induces apoptosis. Unfortunately, we did not observe any cell apoptosis. These results were consistent with those of a previous study on RCAS1 and cell apoptosis (6); namely that RCAS1 does not induce the apoptosis of naïve $\mathrm{T}$ cells. Of note, we observed a significant difference in cell apoptosis between the Ad-RCAS1 group and the Ad-LacZ or control group after the cells were stimulated with IL-2 and PHA. We also observed an increased number of apoptotic cells in the activated $\mathrm{CD}^{+} \mathrm{T}$ cells transfected with Ad-RCAS1 and the percentage of Annexin V-positive cells was $>90 \%$. These results suggested that activated $\mathrm{T}$ cells and $\mathrm{CD}^{+}{ }^{+} \mathrm{T}$ cells overexpressed RCAS1 following transfection with RCAS1, leading to the induction of apoptosis. In the activated $\mathrm{CD}^{+} \mathrm{T}$ cells, the high number of apoptotic cells $(>90 \%)$ may be due to MACS.

Furthermore, using the same method, we also detected the effects of RCAS1 on the apoptosis of immune cells derived from leukemia cell lines, such as Jurkat, K562, THP1 and E003 cells. We observed an increased apoptosis of K562 cells in the RCAS1 group compared with the other groups. However, RCAS1 had no effect on the apoptosis of THP1 and E003 cells, and a low number of apoptotic cells was observed in the Jurkat cells transfected with RCAS1. Following treatment with PHA, the overexpression of RCAS1 significantly increased the apoptosis of Jurkat cells. In order to elucidate the mechanism behind these phenomenon, we examined whether the receptor expression differed in these cells. In our previous study, we induced the expression and purification of GST-RCAS1 fusion protein (11); in this study, we used this GST-RCAS1 fusion protein to detect RCAS1R expression in these cells. Of note, RCAS1R expression was detected in the K562 cells, but low expression levels of RCAS1R were detected in the THP1, E003 and Jurkat cells. RCAS1 expression in the Jurkat cells increased significantly following stimulation with PHA. These results suggest that RCAS1 does not induce the apoptosis of THP1, E003 and Jurkat cells which do not express RCAS1R; however, RCAS1 induces the apoptosis of K562 and PHA-treated Jurkat cells which express RCAS1R. Thus, RCAS1 may be a possible mechanism involved in the induction of cell apoptosis by binding to RCAS1R. The other mechanisms involved remain to be elucidated.

We also used MTT assay to examine the inhibitory effect of RCAS1 on the proliferation of K562 and Jurkat cells. In this study, there was a significant difference in cell viability between the RCAS1 group and other groups in a MOI-dependent manner, indicating that RCAS1 inhibited K562 and Jurkat cell growth in vitro. This result were consistent with the result of cell apoptosis.

Consisting of 2 isoforms, GSK $3 \alpha$ and GSK $3 \beta$, GSK 3 was originally considered to be inexorably linked to glycogen metabolism $(16,17)$. Increasing knowledge has altered the status of GSK3 $\beta$ to that of a broadly influential enzyme that is a crucial regulator of a nubmer of cellular functions, including cellular structure, growth, motility and apoptosis (18). GSK3 $\beta$ activity was significantly reduced by the phosphorylation of the N-terminal serine, Ser9, in GSK3 $\beta$, and Ser21 in GSK3 $\alpha$.
One of the isoforms, GSK3 $\beta$, has been reported to be crucial to cell survival, including the attenuation of apoptosis and NF- $\kappa$ B activation (19). GSK3 regulates Toll-like receptor (TLR)-mediated inflammatory response by differentially affecting the nuclear amounts of the transcription factors, $\mathrm{NF}-\kappa \mathrm{B}$ subunit p65 and cAMP response element-binding protein (CREB), interacting with the co-activator CREBbinding protein (CBP) (20). In this study, we detected GSK3 $\beta$ activity in the K562 cells transfected with Ad-RCAS1 and Ad-LacZ. The upregulation of phGSK3 $\beta$ was detected in the K562 cells transfected with RCAS1, whereas total GSK3 $\beta$ downregulated, suggesting that the overexpression of RCAS1 results in the phosphorylation of GSK $3 \beta$ by a certain pathway. Co-immunoprecipitation assay showed that RCAS1 did not directly bind to GSK $3 \beta$, indicating that RCAS1 possibly interacted with the protein located upstream of GSK3 3 . The inhibition of the GSK3 $\beta$ activity and thus the inhibiton of the $\mathrm{NF}-\kappa \mathrm{B}$ signaling pathway may be involved in the apoptosis of K562 cells induced by RCAS1, although the precise mechanism involved needs to be further elucidated.

A number of previous studies have linked the exposure to RCAS1 as an important factor associated with cancer $(21,22)$. We used immunohistochemical analysis to evaluate RCAS1 expression in breast tissues. RCAS1 expression was observed in the breast cancer tissues, but not in normal breast tissue, suggesting that RCAS1 expression is an important event associated with cancer.

In conclusion, we demonstrate that RCAS1 induces the apoptosis of activated T cells, K562 cells and PHA-activated Jurkat cells. The possible mechanism involved may be that the expression of RCAS1R can be induced. RCAS1 inhibits the growth of Jurkat and K562 cells, and is expressed in breast cancer tissues. In addition, the downregulation of GSK $3 \beta$ may play a certain role in the cell growth inhibition by RCAS1. Our data provide insight into the mechanism through which tumor cells escape from immune surveillance and provide a strategy for the therapeutic intervention of cancer.

\section{Acknowledgments}

This study was supported by grants from the National Natural Science Foundation of China (nos. 30100163 and 30328011), and the National Key Basic Research Program of China (2004CB518802).

\section{References}

1. Tsuchiya $\mathrm{F}$, Ikeda $\mathrm{K}$, Tsutsumi $\mathrm{O}$, et al: Molecular cloning and characterization of mouse EBAG9, homolog of a human cancer associated surface antigen: expression and regulation by estrogen. Biochem Biophys Res Commun 284: 2-10, 2001.

2. Suzuki T, Inoue S, Kawabata W, et al: EBAG9/RCAS1 in human breast carcinoma: a possible factor in endocrine-immune interactions. Br J Cancer 85: 1731-1737, 2001.

3. Akahira JI, Aoki M, Suzuki T, et al: Expression of EBAG9/ RCAS1 is associated with advanced disease in human epithelial ovarian cancer. Br J Cancer 90: 2197-2202, 2004.

4. Takahashi S, Urano T, Tsuchiya F, et al: EBAG9/RCAS1 expression and its prognostic significance in prostatic cancer. Int $\mathrm{J}$ Cancer 106: 310-315, 2003.

5. Aoki T, Inoue S, Imamura H, et al: EBAG9/RCAS1 expression in hepatocellular carcinoma: correlation with tumour dedifferentiation and proliferation. Eur J Cancer 39: 1552-1561, 2003. 
6. Nakashima M, Sonoda K and Watanabe T: Inhibition of cell growth and induction of apoptotic cell death by the human tumor-associated antigen RCAS1. Nat Med 5: 938-942, 1999.

7. Matsushima T, Nakashima M, Oshima K, et al: Receptor binding cancer antigen expressed on SiSo cells, a novel regulator of apoptosis of erythroid progenitor cells. Blood 98: 313-321, 2001.

8. Sonoda K, Miyamoto S, Yamazaki A, Kobayashi H, Nakashima M, Mekada E and Wake N: Biologic significance of receptor-binding cancer antigen expressed on SiSo cells (RCAS1) as a pivotal regulator of tumor growth through angiogenesis in human uterine cancer. Cancer 110: 1979-1990, 2007.

9. Oshikiri T, Miyamoto M, Morita T, et al: Tumor-associated antigen recognized by the 22-1-1 monoclonal antibody encourages colorectal cancer progression under the scanty $\mathrm{CD} 8^{+}$ T cells. Clin Cancer Res 12: 411-416, 2006

10. Chatterjee M, Mohapatra S, Ionan A, et al: Diagnostic markers of ovarian cancer by high-throughput antigen cloning and detection on arrays. Cancer Res 66: 1181-1190, 2006.

11. Hong XJ, Shen FP and Wang QQ: Construction of recombinant GST-RCAS1 fusion gene and its expression in E. Coli. Zhejiang Da Xue Xue Bao Yi Xue Ban 35: 377-383, 2006 (In Chinese).

12. Hong X, Liu Y, Hu G, et al: EBAG9 inducing hyporesponsiveness of $\mathrm{T}$ cells promotes tumor growth and metastasis in 4T1 murine mammary carcinoma. Cancer Sci 100: 961-969, 2009.

13. Reed LJ and Muench HA: A simple method for estimating fifty percent endpoint. Am J Hyg 27 : 493-497, 1938.

14. Engelsberg A, Hermosilla R, Karsten U, Schülein R, Dörken B, and Rehm A: The Golgi protein RCAS1 controls cell surface expression of tumor-associated O-linked glycan antigens. J Biol Chem 278: 22998-23007, 2003.
15. Reimer TA, Anagnostopoulos I, Erdmann B, et al: Reevaluation of the 22-1-1 antibody and its putative antigen, EBAG9/RCAS1, as a tumor marker. BMC Cancer 5: 47, 2005.

16. Embi N, Rylatt DB and Cohen P: Glycogen synthase kinase-3 from rabbit skeletal muscle. Separation from cyclic-AMPdependent protein kinase and phosphorylase kinase. Eur J Biochem 107: 519-527, 1980.

17. Woodgett JR and Cohen P: Multisite phosphorylation of glycogen synthase. Molecular basis for the substrate specificity of glycogen synthase kinase-3 and casein kinase-II (glycogen synthase kinase-5). Biochim Biophys Acta 788: 339-347, 1984.

18. Jope RS and Johnson GV: The glamour and gloom of glycogen synthase kinase-3. Trends Biochem Sci 29: 95-102, 2004.

19. Hoeflich KP, Luo J, Rubie EA, Tsao MS, Jin O and Woodgett JR: Requirement for glycogen synthase kinase-3beta in cell survival and NF-kappaB activation. Nature 406: 86-90, 2000.

20. Martin M, Rehani K, Jope RS and Michalek SM: Toll-like receptor-mediated cytokine production is differentially regulated by glycogen synthase kinase 3. Nat Immunol 6: 777-784, 2005.

21. Dutsch-Wicherek M and Wicherek L: The association of RCAS1 serum concentration with the reversibility or irreversibility of the process of immune cytotoxic activity restriction during normal menstrual cycle, cancer relapse, and surgical treatment for various types of squamous cell carcinomas and adenocarcinomas. Am J Reprod Immunol 59: 266-275, 2008.

22. Dutsch-Wicherek M, Tomaszewska R, Lazar A, Wicherek L and Skladzien J: The association between RCAS1 expression in laryngeal and pharyngeal cancer and its healthy stroma with cancer relapse. BMC Cancer 28: 9-35, 2009. 